\title{
Developing a Scoring System to Determine Necessity and Timing of Surgical Intervention in the Treatment of Cellulitis
}

\author{
Narayana Subramaniam*, Bharati V. Hiremath, Balakrishna M. Naik \\ Department of General Surgery, M.S. Ramaiah Medical College and Hospital, Bangalore, India
}

Email address:

narayana.subramaniam@gmail.com (N. Subramaniam)

To cite this article:

Narayana Subramaniam, Bharati V. Hiremath, Balakrishna M. Naik. Developing a Scoring System to Determine Necessity and Timing of Surgical Intervention in the Treatment of Cellulitis. Journal of Surgery. Vol. 3, No. 2, 2015, pp. 8-11. doi: 10.11648/j.js.20150302.11

\begin{abstract}
Aim: To develop a clinico-pathological scoring system to determine necessity and timing of surgical intervention in cellulitis. Introduction: Cellulitis is a major cause of morbidity, however standard plan of care and treatment guidelines are far from standardized. Our study was done to determine the clinical and pathological markers indicating the need for surgical intervention and derivation of a scoring system. Materials and methods: Cross-sectional prospective observational study of 148 patients of cellulitis presenting to M.S. Ramaiah Hospitals between Jan. 2012 and 2014. Based on clinical judgement they were divided into two groups - those who required surgical intervention and those who did not. All cases were evaluated in terms of clinical and pathological characteristics. These parameters were compared in the two groups and the clinical and laboratory findings that were altered to a degree of statistical significance in those undergoing surgical intervention were noted; the relative risk ratio for surgical intervention of each was taken as a component of the final scoring system. Results: The physical and biochemical parameters found to have a statistically significant correlation with surgical intervention - percentage of area involved $(p<0.001)$, skin necrosis $(p<0.001)$, stretch pain $(p<0.001)$, chronic kidney disease $(p<0.002)$, diabetes mellitus $(p<0.002)$, hyponatremia $(p<0.006)$ and degree of elevation in total count $(p<0.001)$. These were used to derive the scoring system. Low risk was $<5$ points and high risk $>10$ points. Conclusion: This scoring system may be helpful to determine necessity and timing of surgical intervention in cellulitis, especially when to end trial of conservative management. Additionally it may provide risk stratification for prognostic value.
\end{abstract}

Keywords: Cellulitis, Surgery, Scoring System

\section{Background}

Cellulitis is a very common problem encountered all over the world, representing a major cause of morbidity and occasionally even mortality ${ }^{1}$. However standard plan of care and treatment guidelines are far from uniform and are a frequent cause of confusion, worsened by the fact that these patients may be seen by a variety of doctors - general practitioners, physicians, surgeons, paediatricians or dermatologists.

Although there is no dearth of scoring indices and risk stratifications available for determining severity, need for hospitalization and other parameters, as per our knowledge there is no scoring system to determine need for surgical intervention in cellulitis. This decision has always been considered a clinical judgement call, but studies have shown these decisions are often erroneous, resulting in additional patient morbidity, necessitating more objective criteria for determining need for surgery in these patients ${ }^{2}$.

Indications for surgical intervention are highly inconsistent The most cited indication for surgical intervention is the patient having multi-organ dysfunction as a result of severe cellulitis $^{3}$. Other indications include secondary bone or joint involvement, tissue necrosis, involvement of the head and neck area, compartment syndrome and predisposing conditions like abscesses ${ }^{4}$. Failure of response to antibiotics, with a trial of conservative therapy lasting between 12-72 hours was also an indication for surgical intervention, with most interventions being fasciotomies, debridements or incisions and drainages ${ }^{5}$. Many of these factors are evaluated subjectively and hence, no uniform guidelines exist regarding timing of surgical intervention ${ }^{6}$.

Our study was done to determine the clinical and pathological parameters most often associated with surgical 
intervention in order to yield a scoring system to ascertain if and when to operate.

\section{Objectives of the Study}

1. To determine the clinical and pathological parameters most strongly associated with patients of cellulitis undergoing surgical intervention based on prevailing clinical guidelines, as per current standard of care

2. To use them derive a scoring system to determine surgical intervention

\section{Materials and Methods}

\subsection{Study Design and Patient Selection}

The study was a cross-sectional case control study conducted during a period of two years, from January 2012 and January 2014, of 148 in-patients at M.S. Ramaiah Hospital Bangalore, diagnosed to have cellulitis. The diagnosis was made clinically, or by soft tissue ultrasound when in doubt. Based on clinical judgement, this group was divided into two groups - one group of 74 patients who were determined to need surgical intervention and the second group included 74 patients who underwent non-operative management.

In this study, a group of five experienced surgeons (over ten years of experience each) were involved in making the decision regarding surgical intervention. The only absolute indication for surgical intervention that all surgeons agreed on was clinical suspicion of compartment syndrome. Corresponding signs included pallor, reduced peripheral pulses, stretch pain, poikilothermia, paraesthesia, paresis and tissue necrosis. There were no other absolute indications that the surgeons agreed on. Laboratory investigations were not considered while dividing patients into these two groups.

Patients who were initially considered for conservative management, which the patient failed to respond to, later requiring surgical intervention, were ultimately considered in the operative group. The final grouping of patients into surgically-managed and medically-managed was only after completion of treatment.

All patients over the age of 20 years were included and those with necrotizing fasciitis were excluded. Full informed consent was taken from the patients and ethical clearance was issued by the institutional ethical review board.

\subsection{Patient Evaluation}

Patients were evaluated on the basis of local clinical criteria (such as anatomical site, area of cellulitis, tenderness, local rise of temperature, oedema, fluctuance, erythema, crepitus, presence of necrosis, presence of peripheral pulses, joint involvement, stretch pain and calf tenderness)

Systemic clinical criteria were as follows:

- Fever: $\geq 38^{\circ} \mathrm{C}$ core body temperature

- Hypotension: systolic blood pressure $\leq 90 \mathrm{~mm} \mathrm{Hg}$ or patient requiring inotropic support or mean arterial pressure $\leq 60 \mathrm{~mm} \mathrm{Hg}$
- Jaundice: patient clinically icteric, usually corresponding to a serum total bilirubin of $2 \mathrm{mg} \%$

- Respiratory distress: patient having respiratory rate consistently over 20 cycles/min with $\mathrm{SpO}_{2}<92 \%$ on room air or central cyanosis

- Bleeding diathesis: easy bruising, with presence of abnormal bleeding in the form of hematuria, petechiae, malaena, etc

Laboratory parameters

- Haemoglobin: reduced according to age and gender matched average

- Serum albumin $<4 \mathrm{~g} \%$

- Serum sodium $<130 \mathrm{mg} \%$

- Serum creatinine $<1 \mathrm{mg} \%$ in women, $<1.3 \mathrm{mg} \%$ in men

- Total leukocyte count was divided into four subgroups, normal $\left(<12,000 / \mathrm{mm}^{3}\right), 12,000-16,000 / \mathrm{mm}^{3}, 16,000$ $20,000 / \mathrm{mm}^{3}$ and $>20,000 / \mathrm{mm}^{3}$

- Hypoglycaemia $(<60 \mathrm{mg} \%)$ and hyperglycemia ( $\geq 126 \mathrm{mg} \%$ fasting blood glucose or $\geq 200 \mathrm{mg} \%$ random blood glucose)

- Coagulation parameters (prothrombin time $>17 \mathrm{~s}$, aPTT $>34$ s and INR $>1.2$ )

- Liver function tests (any derangement signifying a specific pattern of disease, eg hepatitis, cholestasis)

- Serum procalcitonin $(\geq 2 \mu \mathrm{g} / \mathrm{L})$

- Arterial blood gas (any derangement signifying a specific pattern of respiratory failure with or without compensation)

\subsection{Treatment}

These patients were treated for cellulitis as prevailing standard of care, with no alteration in treatment based on the study by the investigators. The decision to operate was taken by the treating surgeons as per their clinical judgement as mentioned above. The cases were followed up till discharge. Surgery performed was most commonly fasciotomy or debridement. Repeated debridements or extensions of fasciotomy were done as and when required.

The antibiotic policy in our hospital, based on the spectrum of infections we commonly encounter, is as follows:

- Mild cases: Amoxicillin-clavulanic acid \pm clindamycin (based on likelihood of co-infection with anaerobic organisms)

- Intermediate severity: Linezolid \pm clindamycin (based on likelihood of co-infection with anaerobic organisms).

- Severe cases: Pipericillin-tazobactam/meropenem

\subsection{Statistical Analysis}

Descriptive statistical methods were applied. Chi square test/Fisher extract test was applied to the two groups operative and non-operative management groups - to determine the statistical significance of each of the study parameters in the two groups, and yield a relative risk of each of the parameters for surgical intervention, first via a univariate and then a multi-variate analysis. The relative risk of these parameters was the basis for constructing the severity 
score for surgical intervention. Multiple regression analysis was used for comparison of independent variables. The score was then self-validated using the subjects of the study.

\section{Results}

Patients' ages ranged between 20 and 92 years, with majority $(70 \%$ of the conservative management group and $68 \%$ of the operative management group) were between 31 and 60 years of age. There was no significant increase in operative management with advancing age.

Fever was noted in almost two-thirds of the patients. The most common co-morbid associations in operated patients were renal dysfunction (47\%), followed by coagulopathy (18\%), hepatic dysfunction (17\%) and septic shock (13\%). The most common bacteria isolated from those operated were enterobacteriaceae (35\%), streptococci (30\%), staphylococci
(15\%), pseudomonas (5\%) and enterococci (5\%). These were comparable to other available literature ${ }^{7}$.

The lower limb was the most common site for cellulitis $(88.9 \%)$. The area of the region involved by cellulitis was also significantly related to surgical intervention involvement of over $30 \%$ of the region by cellulitis had a strong correlation with surgical management $(p<0.001 *)$. This may be a reflection of the virulence of the organism, the nature of the infection and possibly a poor immune reaction resulting in an inability to control the infection.

The local factors strongly associated with surgical intervention were presence of necrosis and stretch pain. Also significantly associated with surgical intervention were presence of co-morbidities $(\mathrm{p}<0.002 *)$, hyponatremia $\left(\mathrm{P}<0.006^{*}\right)$ and degree of leucocytosis $\left(\mathrm{p}<0.001^{*}\right)$.

The final scoring system is as follows:

Cellulitis severity scoring system for surgical intervention

Presence of stretch pain
Presence of skin necrosis
Percentage of region involved by cellulitis $30-50 \%$
Percentage of region involved by cellulitis $50-70 \%$
Percentage of region involved by cellulitis $>70 \%$
Presence of chronic kidney disease
Presence of diabetes mellitus
Presence of hyponatremia
Total count $16,000-20,000$
Total count $>20,000$
Failure of conservative management after 48 hours (persistent fever, no significant change in
total count, no local response to conservative management)
MAXIMUM POSSIBLE SCORE: 30
Risk stratification:

\begin{tabular}{l}
8 \\
9 \\
2 \\
3 \\
4 \\
2 \\
2 \\
2 \\
2 \\
3 \\
3 \\
Unlikely to require surgical intervention $<5$ points \\
Highly likely to require surgical intervention $>10$ points \\
\hline
\end{tabular}

\section{Discussion}

The term cellulitis, in clinical practice, can often refer to a wide spectrum, from an indolent condition that doesn't require admission to a life-threatening illness causing multiple organ dysfunctions and necessitating aggressive medical and surgical intervention to prevent mortality. Very often, the severity of the condition is underestimated initially ${ }^{8}$ and the need for surgical intervention is overlooked, resulting in a delay in definitive treatment and an increase in morbidity and possibly mortality 9 Additionally, guidelines for early signs or predictors for surgical intervention (before the onset of compartment syndrome and/or frank tissue necrosis) are lacking ${ }^{10}$. As per our knowledge, no scoring system exists for determining surgical intervention in cellulitis.

Our study design is based on the current standard of care for cellulitis - determining the need for surgical intervention is largely a clinical decision without much objective data and hardly any consensus, except in the presence of unequivocal features of compartment syndrome. The idea of our study was to try and derive an objective scoring system that would take into consideration the parameters that were strongly associated with surgical intervention that were not obvious at a first glance (necrosis, stretch pain, signs of limb ischemia) and were not previously agreed upon indicators that surgery was necessary.

As with all clinical scoring systems, this is not meant to be absolute - it is not of much significance of those patients with very mild or very severe cellulitis in whom the role of surgical intervention is clear; it is of most value in those of intermediate severity, especially those not responding satisfactorily to conservative management, since the scoring system represents those factors most strongly associated with a need for eventual surgical intervention.

We found that by use of our scoring system, 20 patients $(27 \%)$ in the group that required surgical intervention had no unequivocal local signs that necessitated surgery (as per the clinical consensus reached by our surgical team), even though none of them were deemed to have undergone unnecessary surgeries. We interpreted this to mean that clinical determination of patient's need for surgery was likely to be influenced by other factors, such as duration of symptoms, presence of co-morbidities and presence of signs of organ dysfunction - none of which the surgeons had mentioned in their clinical consensus. This $27 \%$ is likely to represent a combination of those factors.

The average score of patients who underwent surgical intervention was 17 points. However no patients who 
underwent surgery were found to have a score of less than 11, which is why we our risk stratification places any score above 10 points likely to require surgical intervention. The two most heavily weighted factors on the scoring system are stretch pain ( 8 points) and skin necrosis ( 9 points), which are not individually adequate to label a patient high risk. The reason is simple - in our 74 patients, when present together, the specificity for compartment syndrome was $100 \%$, but individually the specificities were $77 \%$ and $82 \%$ respectively. This is because of other differential diagnoses that can produce these findings (eg. deep vein thrombosis, arthritis, dermatological disorders, etc.)

In our self-validation of the score, we did not find that any patients with a high score who had been treated successfully on conservative management - implying that our score is unlikely to propose surgery where unnecessary, though validation is required with a larger sample size. Conversely there were no false negatives (no patients having a score less than five points on admission subsequently required surgery). Additionally, as with other similar scoring systems, the most popular being the LRINEC for necrotizing fasciitis, this score is most useful for early detection of severe cellulitis and is of very limited value for mild infections ${ }^{11}$, our focus being on early predictors of severe disease and need for surgical intervention.

The number of deaths in the two groups were $1(1.35 \%)$ in the non-surgical group and $6(8.1 \%)$ in the surgical group, which was found to be statistically significant $\left(\mathrm{p}<0.001^{*}\right)$. Moreover it was found that 5 out of 6 patients who died in the surgical group (83.33\%) had a cellulitis severity score $\geq 21$ points. We also noted that only 1 patient in the surgical group with a score of higher than 21 points survived. Hence, as tempting as it seems, whether this figure can be used as prognostic indicator is unknown due to the limited sample size.

There were 5 patients who were initially managed conservatively, who later required surgery after they failed to respond. Interestingly, based on the calculated cellulitis severity scoring on admission, 4 patients $(80 \%)$ were in the high risk category ( $>10$ points), which means that the score may be of value in determining those unlikely to respond to conservative management and have a high likelihood of requiring surgery. An increase in the score, which can be done at 24 hour intervals to assess response to conservative management, can also be considered failure of medical management and an indication to operate. Also, 4 patients required multiple re-debridements (on average 3 surgeries). However there was no pattern noted in their severity scoring that distinguished them from those who underwent only one surgery.
This scoring system, we believe, may help fulfil an unmet need to determine severity of illness at presentation and outline further management. The role of predicting the need for surgical intervention is two-fold - for non-surgeons managing cases of cellulitis it represents the need for seeking surgical care, especially important if it involves referring the patient to a higher centre for treatment and for surgeons who are unclear on the need for surgical intervention, especially in a background of poor response to conservative management.

\section{References}

[1] Stevens DL, Bisno AL, Chambers HF, Everett ED, Dellinger P, Goldstein EJ, et al. Practice guidelines for the diagnosis and management of skin and soft-tissue infections. Clin Infect Dis. 2005 Nov 15;41:1373-406.

[2] DeFrances CJ, Lucas CA, Buie VC, Golosinskiy A. 2006 National Hospital Discharge Survey. National health statistics reports; no 5. Hyattsville, MD: National Center for Health Statistics; 2008

[3] Cox NH, Colver GB, Paterson WD. Management and morbidity of cellulitis of the leg. J R Soc Med. 1998 Dec;91:634-7.

[4] Morpeth SC, Chambers ST, Gallagher K, Frampton C, Pithie AD. Lower limb cellulitis: Features associated with length of hospital stay. J Infect. 2006 Jan;52:23-9

[5] Dong SL, Kelly KD, Oland RC, Holroyd BR, Rowe BH. ED management of cellulitis: A review of five urban centers. Am J Emerg Med. 2001 Nov;19:535-40.

[6] Börnsdóttir S, Gottfredsson M, Thórisdóttir AS, Gunnarsson GB, Ríkardsdóttir H, Kristjánsson M, et al. Risk factors for acute cellulitis of the lower limb: a prospective case-control study. Clin Infect Dis. Nov 15 2005;41:1416-22

[7] Gabillot-Carré M, Roujeau JC. Acute bacterial skin infections and cellulitis. Curr Opin Infect Dis. Apr 2007;20:118-23

[8] Vinh DC, Embil JM. Rapidly progressive soft tissue infections. Lancet Infect Dis. 2005 Aug;5:501-13

[9] Carratala J, Roson B, Fernandez-Sabe N, Shaw E, del Rio O, Rivera A, et al. Factors associated with complications and mortality in adult patients hospitalized for infectious cellulitis. Eur J Clin Microbiol Infect Dis. 2003 Mar;22:151-7

[10] Ellis Simonsen SM, van Orman ER, Hatch BE, et al. Cellulitis incidence in a defined population. Epidemiol Infect. Apr 2006;134:293-9

[11] Wong CH, Khin LW, Heng KS, Tan KC, Low CO. The LRINEC (Laboratory Risk Indicator for Necrotizing Fasciitis) score: a tool for distinguishing necrotizing fasciitis from other soft tissue infections. Crit Care Med 2004;32:1535-41. 